\title{
Diffraction Limited Near Infrared Imaging of the Central Parsec of the Galaxy
}

\author{
Eckart, A., Genzel, R., Hofmann, R., Sams, B.J., Tacconi-Garman, L.E. \\ Max-Planck Institut für extraterrestrische Physik, 8046 Garching, Germany
}

\begin{abstract}
We present deep 1.6 and $2.2 \mu \mathrm{m}$ images of the central parsec of the Galaxy at a resolution of $0.15 "$ ". Most of the flux in earlier seeing limited images comes from about 340 unresolved stellar sources with $K \leq 14$. The IRS 16 and 13 complexes are resolved into about two dozen and half a dozen sources, a number of which are probably luminous hot stars. We confirm the presence of a blue near infrared object $(K \approx 13)$ at the position of the compact radio source $\mathrm{Sgr} \mathrm{A}^{*}$. The spatial centroid of the source number distribution is consistent with the position of Sgr A* but not with a position in the IRS 16 complex. The stellar surface density in the central 10" is very well fitted by an isothermal cluster model with a well defined core radius. The derived core radius of all 340 sources is $0.15 \pm 0.05 \mathrm{pc}$. The central stellar density is a few times $10^{7} \mathrm{M}_{\odot} \mathrm{pc}^{-3}$. Buildup of massive stars by merging of lower mass stars and collisional disruption of giant atmospheres are very probable processes in the central $0.2 \mathrm{pc}$.
\end{abstract}

\section{Introduction}

For the last half dozen years infrared array detectors have been available for seeing limited $(\leq 1$ ") near-infrared imaging of the Galactic center (e.g. Forrest, Pipher and Stein 1986, Tollestrup, Capps and Becklin 1989, Rieke, Rieke and Paul 1989, DePoy and Sharp 1991). We report the results of diffraction limited near-infrared observations $\left(0.15^{\prime \prime}\right.$ or $6.2 \times 10^{-3} \mathrm{pc}$ at $\left.8.5 \mathrm{kpc}\right)$ - of the central parsec with the MPE speckle camera SHARP. These Images improve upon the first Galactic center SHARP data of Eckart et al. (1992). A more detailed discussion is presented in a forthcoming paper (Eckart et al. 1993).

\section{Observation and Data Reduction}

In March 1992 and August 1992 we employed the new MPE infrared high resolution camera, SHARP, at the New Technology Telescope (NTT) of the European Southern Observatory (ESO) in La Silla, Chile, for further near-diffraction limited imaging (0.05" per pixel; integration times of 0.3 and $1 \mathrm{sec}$ per frame) at $\mathrm{H}(1.6 \mu \mathrm{m})$ and $\mathrm{K}(2.2 \mu \mathrm{m})$ of the central $\approx 20$ " diameter region. SHARP uses a $256^{2}$ pixel NICMOS III detector array manufactured by Rockwell International Corporation. The frames were corrected for flat field and dead pixels. After image selection based on seeing estimates a simple shift-and-add algorithm was applied which produced diffraction limited images on top of a seeing background starting at the $30 \%$ peak intensity level. The images "CLEANed" from this background with their average shift-and-add reference point spread function (IRS 7/ IRS 16NE) using a Lucy algorithm (Lucy 1974) and then reconvolved with a Gaussian of 0.15 " FWHM. 


\section{Results and Discussion}

Fig.1 shows a contour map obtained from K-band speckle data taken in March 1992. For the central part of the image the total integration time is $\approx 4$ hours. The dynamic range is larger than 7 magnitudes with respect to IRS $7(K=6.8)$. The emission is resolved in individual stars and groups of stars. There is no convincing evidence for extended/diffuse $2 \mu \mathrm{m}$ emission. Within the calibration uncertainties 340 compact sources of $\mathrm{K} \leq 14$ contain all the flux density ( $\approx 66 \%$ in the brightest 29 sources). The spatial median of the source number distribution (irrespective of their fluxes) is about 0.6 " $\pm 0.7^{\text {" }}$ $\mathrm{W}$ and 1.3" $\pm 1.0^{\prime \prime} \mathrm{S}$ of Sgr A" (3 $\sigma$ errors), indicating that the cluster center could be on Sgr A* but not in the IRS 16 complex. The stellar density distribution (positions only) is very well fitted by an isothermal cluster model. The best fitting isothermal density distribution (dashed curve in Fig.2) corresponds to a core radius of 3.8 " $(0.15 \mathrm{pc})$ and a central surface density of 3 sources per arcsec ${ }^{2}$. The $r^{-1.8}$ density power law previously determined from the surface brightness distribution (cf. Bailey 1980) does not fit the central few arcseconds. A core radius of $r_{0}=0.15 \pm 0.05 \mathrm{pc}$ and a line-of-sight central velocity dispersion $\sigma_{o}=100 \pm 25 \mathrm{~km} / \mathrm{s}$ (cf. Sellgren et al. 1990) imply a central cluster density of $\rho_{0}=10^{7.7 \pm 0.5} M_{\odot} p c^{-3}$, and a core mass of $M_{\circ}\left(r_{o}\right)=10^{5.7 \pm 0.3} M_{\odot}$. At present there is no compelling evidence for formation of massive stars from self-gravitating gas clouds in the central few parsecs. The very high stellar density would allow formation of massive stars by sequential buildup via collisions and mergers (Lee 1987, 1990). Calculations indicate that this is an attractive mechanism for explaining the presence of stars with masses $>10 \mathrm{M}_{\odot}$ in dense clusters (Lee 1987). We confirm a blue near infrared object $(\mathrm{K} \approx 13)$ at the position of the compact radio source $\mathrm{Sgr} \mathrm{A}^{*}$. Its identification as the near-infrared counterpart of Sgr A* can only be tentative at present. With 2 sources per $\operatorname{arcsec}^{2}$ with $\mathrm{K}<13.7$ the probability of a chance alignment is substantial: about $5 \%$ to $20 \%$ depending on the method of calculation. The blue color $\mathrm{K}$-band source may be a hot foreground cluster member or the scattered radiation from a hot accretion disk around Sgr A*.

Bailey, M.E., 1980, M.N.R.A.S., 190, 217.

DePoy, D.L. and Sharp, N.A., 1991, A.J., 101, 1324.

Eckart, A., Genzel, R., Krabbe, A., Hofmann, R., P.P. van der Werf, Drapatz, S., 1992, Nature, $335,526$.

Eckart, A., Genzel, R., Hofmann, R., Sams, B., Tacconi-Garman, L.E., 1993, Ap.J. (Letters), submitted.

Krabbe, A., Genzel, R., Drapatz, S. and Rotaciuc, V., 1991, Ap. J. (Letters), 382, L19.

Krabbe, A., et al. 1993, in prep.

Forrest, W.J., Pipher, J.L. and Stein, W., 1986, Ap. J. (Letters), 301, L49.

Lee, H.M., 1987, Ap. J., 319, 771.

Lee, H.M., 1990, in Dynamics of Dense Stellar Systems, ed. D. Merritt, Cambridge Univ. Press, p. 105

Lucy, L.B., 1974, A.J., 79, 745.

Rieke, G.H., Rieke, M.J. and Paul, A.E., 1989, Ap. J., 336, 752.

Sellgren et al., 1990, Ap. J., 359, 112.

Tollestrup, E.V., Capps, R.W. and Becklin, E.E., 1989, A.J., 98, 204. 


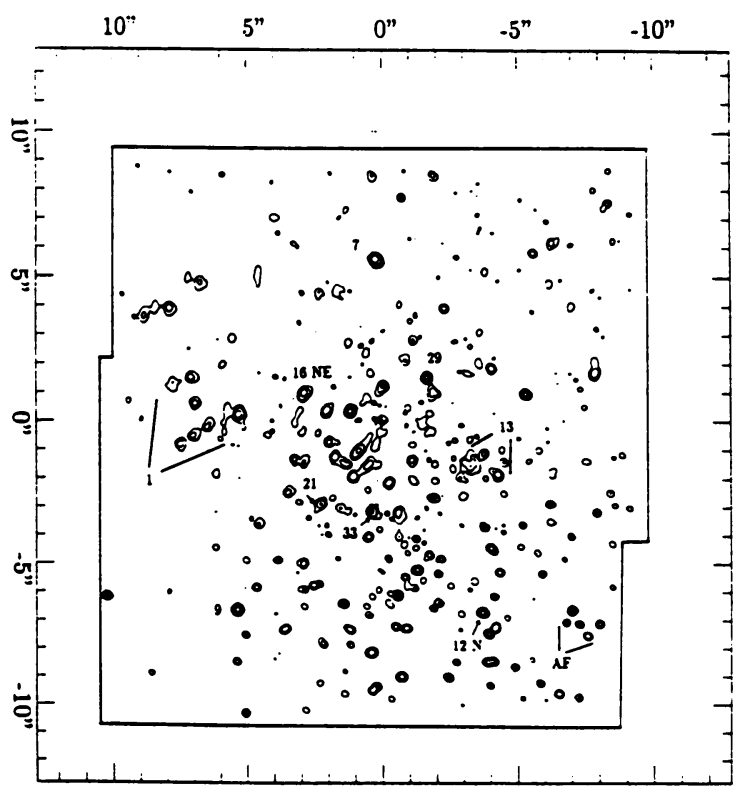

Fig.1: Mosaic of K-band speckle image reconstructions of the central 20 " $\times 20^{\text {" }}$ of the Galaxy at a spatial resolution of $0.15^{n}$ FWHM ( $2.2 \mu \mathrm{m}$ ESO NTT diffraction limit) centered on the position of the compact radio source Sgr $\mathrm{A}^{*}$. Boundaries of the mosaic are indicated by solid lines. Contour levels are 14.5, 12.5, 10.5, 8.5, 6.8 magnitudes. The cross indicates the position of Sgr $A^{*}$

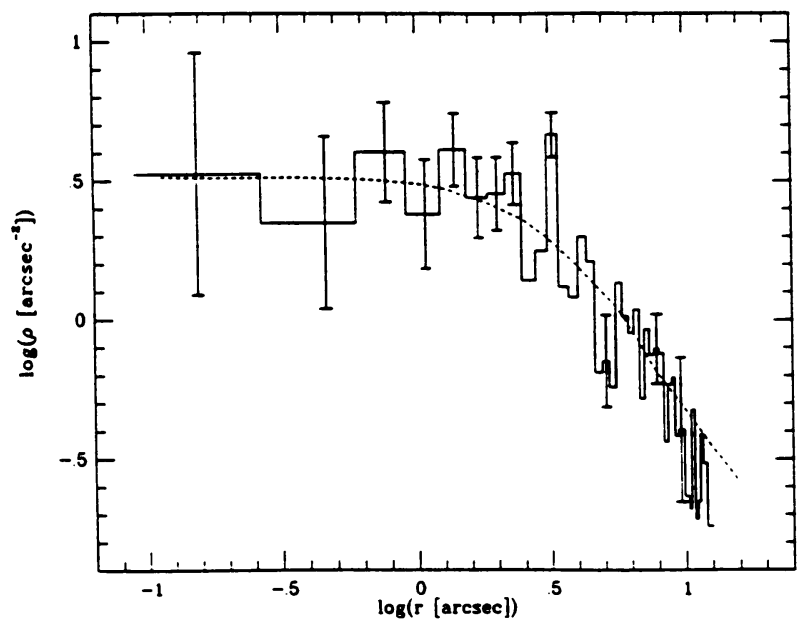

Fig.2: Surface density $\rho$ in sources per $(\operatorname{arcsec})^{2}$ for all 340 stars with $K<14$ in $0.3^{\text {" wide annuli }}$ of radius $\mathrm{r}$ from the radio position of $\mathrm{Sgr} \mathrm{A}^{*}$ (histogram). Representative error bars indicate the statistical $\left(N^{1 / 2}\right)$ uncertainties in each annulus. The resulting distribution is very well fitted by an isothermal stellar density distribution (dashed curve) corresponding to a core radius of 3.8 " $(0.15 \mathrm{pc})$ and a central surface density of 3 sources per (arcsec $)^{2}$. 